\title{
Smart Tutoring System: A Predictive Personalized Feedback in a Pedagogical Sequence
}

\author{
https://doi.org/10.3991/ijet.v16i20.24783 \\ Fatima-Zohra Hibbi $\left.{ }^{\bowtie}\right)$, Otman Abdoun, Haimoudi El Khatir \\ Abdelmalek Essaâdi University, Larache, Morocco \\ fatima.zohra.officialegmail.com
}

\begin{abstract}
Feedback may be an effective interaction provided by the intelligent tutoring system. Nevertheless, the learning feedback is not easily definable, especially in front of learners with their characteristics and preferences. In this work, the authors propose to predict personalized feedback in a programming language learning context that promotes the feedback of the ITS according to the learner preferences and learner style. The recommended method uses a combination of machine learning techniques to suggest the best appropriate feedback according to learner's preferences and characteristics. For that purpose, the predictive personalized feedback method will respect the following phases: collect the learning experience from the learning resources (LR) and learner preferences (LP), generate groups of clusters that contain the common characteristics using the k-means algorithm, and define the association rules between the four categories and their corresponding activity. Finally, generate the personalized feedback and propose the recommendation through the intervention of an expert in the field.
\end{abstract}

Keywords-Intelligent tutoring System, feedback, Machine Learning

\section{Introduction \& Background}

Feedback is an essential form of an intelligent tutoring system intervention. The feedback can help students to identify and correct their mistakes, support learners to correct their knowledge and acquire new knowledge in a learning sequence [1]. Several forms of feedback can be used by the tutor in the intelligent tutoring system; such as the tutor can give immediate feedback, or illustrate the correct answer. On the other hand, the tutor can evaluate the level of learner knowledge or understand their deficiencies [2][3].

Numerous researchers were investigated and studied the problems and improvement of the feedback in the intelligent tutoring system; some researchers apply the dialogical techniques to know the level of understanding the pedagogical sequence [4]. Others are concentrated to analyze the feedback model of the smart tutoring system using learning analytics [5]. Further investigation was focused on the provision of feedback using clustered solution space [6]. 
Some researchers proposed a new intelligent tutoring system named Smart Tutoring System (STS) which combined both tutors: explicit and implicit [8] or to convert tacit knowledge into explicit knowledge [9] to improve the feedback of the tutor. Nevertheless, these feedbacks are note personalized according to the learner preferences and style. The aim of this paper is to personalize the feedback of the tutor in a pedagogical sequence of the smart tutoring system using Machine Learning techniques.

The article is organized as follow: Section 2 presents the proposed method and the description of the five steps. Section 3 gives an overview, the feature selection method and the algorithm of selecting the optimal number of clustering using Elbow method. Finally, section 4 presents the conclusion and insight of upcoming works.

\section{Proposed method: Predictive Personalized feedback in Smart Tutoring System}

In this part, the authors illustrated the proposed method on how machine learning techniques can be used to personalize the feedback of the smart tutoring system in a pedagogical sequence. At the outset, a learning experience is required; it's collected from learning resources and learner preferences.

The learning experience will be clustered to generate $\mathrm{N}$ groups with similar preferences and characteristics. The conception of our proposed method and its element is presented in figure-1-.

Our method consists of four steps: the learning experience, clustering, and association rules. Then, generate the personalized feedback and using the expert to propose the recommendation. Each step in the proposed process is described below:

- Stage 1: Dataset: Learning experience

To define the dataset, the first step aims to generate a learning experience from the learners' resources $(\mathrm{LR})=\{\mathrm{LR} 1, \ldots, \mathrm{LRn})$ and learner preferences $(\mathrm{LP})=$ $\{\mathrm{LP} 1, \ldots, \mathrm{LPn}\}$. The data will be collected from the E-learning platform of the Polydisciplinary faculty, Larache. The form of the LR and LC are listed underneath:

- Type of Attribute: Text, Video, audio, interactive;

- Time: Short, Medium, Long, and Longer;

- Network: Online, offline;

- Period: Morning, Afternoon, Evening, Night;

- Assessment type.

After that, a learner experience is obtained and stored in the learner data to use as a dataset. Afterwards, the authors will detail the second stage.

- Stage 2: Clustering

The objective of this step is to separate the data into homogeneous groups with similar features. In this contribution, we have $\mathrm{N}$ categories, each of them supporting a type of activity. The classification of these categories is based on the type of attribute, the period and the time. 
The authors decided to use the partitioning method with the application of the KMeans algorithm. The choice is not arbitrary; the segmentation of this algorithm is linear in terms of the number of data sets, which increases the execution time. It doesn't take a long time to classify similar characteristics in data like hierarchical algorithms.

- Stage 3: Association rules

In this stage, we will classify the categories defined in the second step into four pedagogical sequence activities: $\mathrm{A}=\{\mathrm{A} 1, \mathrm{~A} 2, \mathrm{~A} 3, \mathrm{~A} 4\}$ using the association rules algorithm. The four divisions are listed below:

- C1: Practical;

- C2: Theoretical;

- C3: Flexional;

- C4: Analytical.

- Stage 4\&5: Personalized and recommended feedback

Step 4 and five are complementary. Step 4 makes the corresponding activity of each category; to propose a list of recommended feedback, the intervention of an expert is required.

The fiftieth step aims to suggest the appropriate activity for the learner taking into account their preferences; then illustrate the recommendation into the inter-face layer of the intelligent tutoring system.

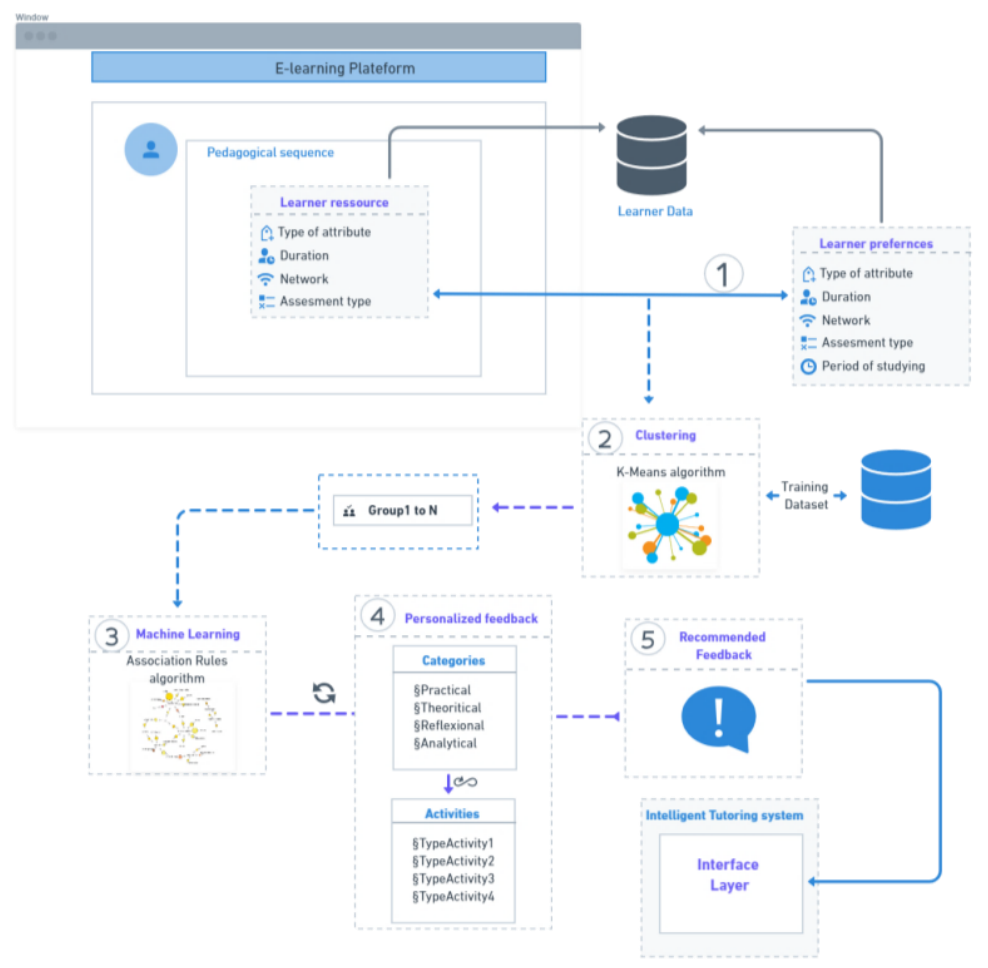

Fig. 1. The process of personalized feedback in the intelligent tutoring system 


\section{Clustering: K-means method}

\subsection{Overview}

Clustering is an unsupervised classification method; its objective is to separate unlabelled data into homogeneous groups with common characteristics [7]. The similarity between two data can be inferred from the "distance" between their descriptors; thus, two very similar data are two data whose descriptors are very close. This definition makes it possible to formulate the problem of data partitioning as the search for $\mathrm{K}$ "prototype data", around which the other data can be grouped.

In this contribution, the authors try to personalize the tutor's feedback based on the learners' choices and preferences during the simulation of a pedagogical sequence. To achieve the objective, the authors propose an overall classification of the activities presented in the pedagogical sequence; a categorization is constructed with similar points and corresponds to specified characteristics. To handle these points, there are several types of clustering.

It is easy to implement k-means and identify unknown data sets from complex data sets. The results are very easy to interpret. K-Means generates cluster descriptions in a minimized form to maximize the understanding of the data.

\subsection{Features Selection}

To select the appropriate features, a Backward Elimination technique is required; it is used to remove those features that do not have a significant effect on the dependent variable. The figure-2- illustrates the process of building the significant features based on the Backward Elimination Features (BEF)

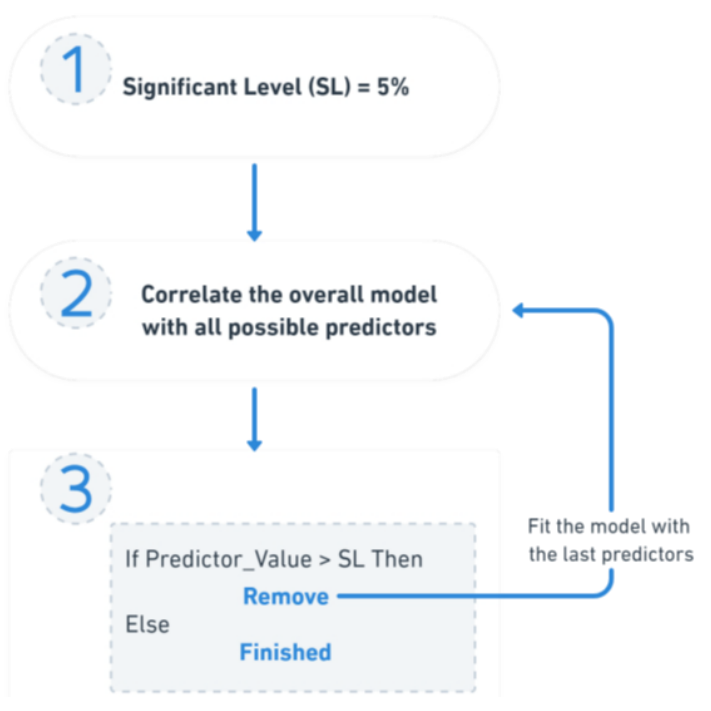

Fig. 2. Backward Elimination Features (BEF) 


\subsection{The optimal number of clusters}

To determine the optimal selection $\mathrm{K}$, a performance data and evaluation clustering quality as needed. There are two methods to find the optimum number of clusters; the first one is the silhouette technique which used the silhouette coefficient, on the other hand, the Elbow method which, is used the original scoring function in the K-means algorithm.

In this contribution, the authors choose to use the Elbow method. This method provides several clusters according to the learners' preferences using the table of features. The table presents the algorithm of selecting the optimal number of clustering using the Elbow method:

Table 1. Algorithm: pseudo-code

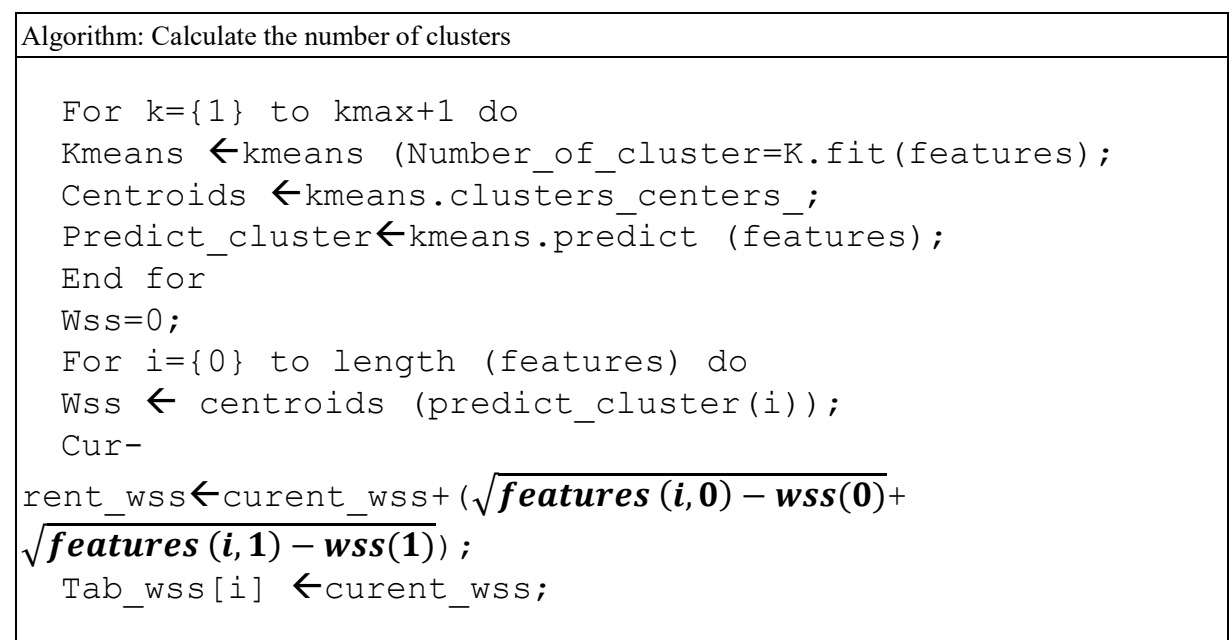

\section{$4 \quad$ Conclusion and future works}

The article presented the previous researchers and the suggested solution on the intelligent tutoring system feedback, proposed a new method to personalize the feedback, and deducted the uses of k-means algorithm as the appropriate method to generate a group of clusters.

This work was carried out to present a new method to personalize feedback in a pedagogical sequence of a programming language learning method. The authors are positive that this new method will conduct to a good prediction of a personalized feedback. Implementing and approving of the effectiveness of these recommendations are lifted as a future works. 


\section{$5 \quad$ References}

[1] Davide Fossati (2008). The role of positive feedback in intelligent tutoring systems. In Proceedings of the 46th Annual Meeting of the Association for Computational Linguistics on Human Language Technologies: Student Research Workshop (HLT-SRWS '08). Association for Computational Linguistics, USA, 31-36. https://doi.org/10.3115/1564154. $\underline{1564162}$

[2] Y. Gong, J.E. Beck and C. Ruiz (2012). Modeling Multiple Distributions of Student Performances to Improve Predictive Accuracy. Book Chapter Lecture Notes in Computer Science, Volume 7379, User Modeling, Adaptation, and Personalization, Pages 102-113. https://doi.org/10.1007/978-3-642-31454-4 9

[3] N.Jones, P.Georghiades and J.Gunson (2012). Student feedback via screen capture digital video: stimulating student's modified action. Higher Education, Volume 64, Number 5, Pages 593-607. https://doi.org/10.1007/s10734-012-9514-7

[4] Dāboliņš, J., \& Grundspeṇķis, J. (2013). The Role of Feedback in Intelligent Tutoring System, Applied Computer Systems, 14(1), 88-93. https://doi.org/10.2478/acss-2013-0011

[5] Hibbi, F.Z., Abdoun, O., Haimoudi, E.K., "Exploration of Analytical Mechanisms in he Feedback model”, Procedia Computer Science, 148, pp. 201-207, 2019. https://doi.org/10. 1016/i.procs.2019.01.037

[6] Gross, S. et al (2012). Feedback Provision Strategies in Intelligent Tutoring Systems Based on Clustered Solution Spaces. DeLFI.

[7] Neha Soni, Amit Ganatra (2012), Comparative study of several Clustering Algorithms, International Journal of Advanced Computer Research (ISSN (print): 2249-7277 ISSN (online): 2277-7970) Volume-2 Number-4 Issue-6.

[8] Hibbi F.Z., Abdoun O., Haimoudi E.K. Integrating an Intelligent Tutoring System into an Adaptive E-Learning Process. In: Dos Santos S., Maslouhi M., Okoudjou K. (eds) Recent Advances in Mathematics and Technology. Applied and Numerical Harmonic Analysis. Birkhäuser, Cham. https://doi.org/10.1007/978-3-030-35202-8 8

[9] Hibbi, F., Abdoun, O., \& El Khatir, H. (2020). Extract Tacit Knowledge in the Learner Model of the Smart Tutoring System. International Journal Of Emerging Technologies In Learning (IJET), 15(04), pp. 235-240. https://doi.org/10.3991/ijet.v15i04.11781

\section{Authors}

Fatima-Zohra Hibbi is a PhD candidate at the Department of Computer Science at Abdelmalek Essadi University. She had her engineering in pedagogy and multimedia. Her research interest includes learning analytics, e-learning and AI in learning.

Otman Abdoun is a professor of computer science at Abdelmalek Essaadi University. His current research includes optimization, problem-solving and learning technology. (E-mail: abdoun.otman@gmail.com)

Haimoudi El Khatir is a professor in the department of computer science in Abdelmalek Essaadi University, Larache, Morocco. (E-mail: elkhatir@gmail.com)

Article submitted 2021-06-14. Resubmitted 2021-07-28. Final acceptance 2021-08-01. Final version published as submitted by the authors. 